\title{
A STUDY ON STAGES OF QUEUING SYSTEM IN AIRCRAFT CONTROL SYSTEM
}

S. Maragathasundari

Associate professor, Department of Mathematics, Kalasalingam Academy of Research and Education.

Krishnankoil, (India).

E-mail: maragatham01@gmail.com ORCID: https://orcid.org/0000-0003-1210-6411

C. Prabhu

Assistant professor, Department of Mathematics, Kalasalingam Academy of Research and Education.

Krishnankoil, (India).

E-mail: cprabhumath4@gmail.com ORCID: https://orcid.org/0000-0003-3879-3299

Manikandan Palanivel

Assistant professor, Department of Electronics and Communication Engineering, Kalasalingam Academy of Research and Education.

Krishnankoil, (India).

E-mail: maanip85@gmail.com ORCID: https://orcid.org/0000-0002-5737-0235

Recepción: 05/12/2019 Aceptación: 15/01/2020 Publicación: 23/03/2020

\section{Citación sugerida:}

Maragathasundari, S., Prabhu, C., y Palanivel, M. (2020). A study on stages of queuing system in aircraft control system. 3C Tecnología. Glosas de innovación aplicadas a la pyme. Edición Especial, Marzo 2020, 91-111. http://doi.org/10.17993/3ctecno.2020.specialissue4.91-111

\section{Suggested citation:}

Maragathasundari, S., Prabhu, C., \& Palanivel, M. (2020). A study on stages of queuing system in aircraft control system. 3C Tecnología. Glosas de innovación aplicadas a la pyme. Edición Especial, Marzo 2020, 91-111. http://doi.org/10.17993/3ctecno.2020.specialissue4.91-111 


\section{ABSTRACT}

This inspection looks into a Non Markovian covering issues in which the organization is rendered in two stages. Customers meet up in bunches seeks after a Poisson assignment. Organization time seeks after a general scattering. After the fulfillment of the second period of organization, the server goes for a required vacation, if in need the server has the decision go take a comprehensive trip. Here vacation in the sense addresses the period in the midst of which the help attempts to be improving the state of the server. In like manner, service blocks carelessly in the midst of the period of organization. Everything considered, conditions, it is unavoidable. In this coating issue, separation occurs in the midst of the second period of organization and acknowledged to occur according to a Poisson stream. Similarly, to dodge the blockage, in the midst of the period of optional expanded vacation, a stand by the server is given. At whatever point the server meddles with, it is sent to fix process quickly promptly. For the above portrayed Queuing model, by strategies for the fortifying variable framework, we get the persisting state results in express and close to respect the probability creating capacities with respect to the amount of customers in the line, the typical number of customers, idle time of the server, use factor and the ordinary holding up time in the line. Numerical portrayal and agrow graphical examination are done toward the end to favor the model.

\section{KEYWORDS}

Non-markovianqueue, Optional extended vacation, Queuing performance measures. 


\section{INTRODUCTION}

Queuing speculation is the numerical examination of holding up lines or Queues. It is a basic piece of Mathematics with associated likelihood, quantifiable spread, examination, matrix theory and complex examination. It furthermore falls under the locale of choice hypothesis. It is a practical circumstance that the server is inaccessible to serve the clients amid intermittent timeframes. The period for which the server is inaccessible to serve the clients as indicated by a known or obscure calendar is characterized to be the server getaway period. In lining, writing, the term get-away alludes to a length in which upkeep work to be completed by the server. Doshi (1986) made a broad review on line frameworks with getaways. Madan (2000) examined two stage heterogeneous administrations with the Bernoulli excursion lining model. The same work was later proposed by Keilson and Servi (1987) under certain modifications. Madan and Abu Al-Rub (2004) contemplates the staged kind server getaways base on single get-away approach. The single entry lining framework M/G/1 have been examined by various creators, as Madan and Baklizi (2002), Artalejo and Choudhury (2004) and so on because of its wide applications.All the more as of late, the vast majority of the investigations have been dedicated to group landing excursion models under various get-away approaches due to its interdisciplinary character. Numerous scientists have considered cluster entry lines with get-away time, weeludethe peruse to Altman and Yechiali (2006), Lee, Lee and Chae (1994). In later years a lot of work has been done on clump entry lines with getaways and arbitrary breakdowns. Maragathasundari and Balamurugan (2015) have considered a cluster entry line of administration in two phases with a Bernoulli plan get-away pursued by an all-encompassing get-away and benefit interference. Maragathasundari and Dhanalakshmi (2018) made a coating approach in Mobile adhoc frameworks. Maragathasundari and Srinivasan (2012) investigated non Markovian feedback queue with multiple server vacation. Multistage cluster arrival queue with service interruption have been well analyzed by Maragathasundari and Srinivasan (2015). Discretionary services are well studied by Maragathasundari and Srinivasan (2017) in a Non Markovian Queue. Maraghi, Madan and Darby-Dowman (2010) made an analysis over second discretionary service 


\section{APPLICATION OF THE MODEL DEFINED}

The queuing model what we implemented here is very much suitable for aircrafts scheduling done to the air traffic controllers. The air traffic controller can serve in the form of first come first serve basis. But the aircrafts what we considered here as the customers are arriving as the batches. The air traffic controller provides various services to maintain safe and secure journey provided to the travelers.

\subsection{AIR TRAFFIC CONTROL SYSTEM}

The errand of guaranteeing safe tasks of business and private flying machine falls on air traffic controllers. They should facilitate the developments of thousands of flying machines, keep them at safe separations from one another, immediate them amid departing and arriving from air terminals guide them around awful climate and guarantee that traffic streams easily with negligible postponements. In this article, we will inspect airport regulation in the United States. We'll pursue a departure from takeoff to landing, taking a gander at the different controllers included, what everyone does, the gear they use and how they are prepared.

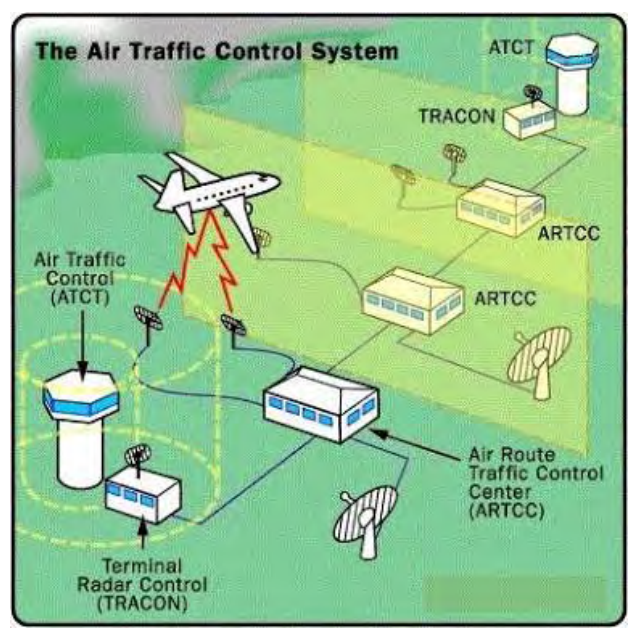

Figure 1. The Air traffic control system.

\subsection{AIR TRAFFIC SERVICES}

Air Traffic Services conveys sheltered, secure, and successful administration for the National Airspace System and worldwide airspace assigned to U.S. control. We are liable for Airport 
Traffic Control Towers (Federal and Contract), Terminal Radar Approach Control offices, Air Route Traffic Control Centers, and Combined Control Facilities to direct flying machine through their different periods of flight. Air traffic controllers at every office are kept up by various specialists, for example, meteorologists, traffic the executive specialists, and $\mathrm{AF}$ administrators who keep up and fix the hardware substructure of the ATC framework.

In this work, we are thinking about ATC, Air Traffic Controller as the server for giving administrations to the arriving Aircrafts. ATC fuses show and control supports, this foundation incorporate $\mathrm{PC}$ frameworks of changing vintages, complex voice and information exchanging gear, radio and microwave transmission frameworks, neighborhood and remote found radio and radar frameworks, and also ecological and electric power molding and reinforcement frameworks, which are required by the hardware.

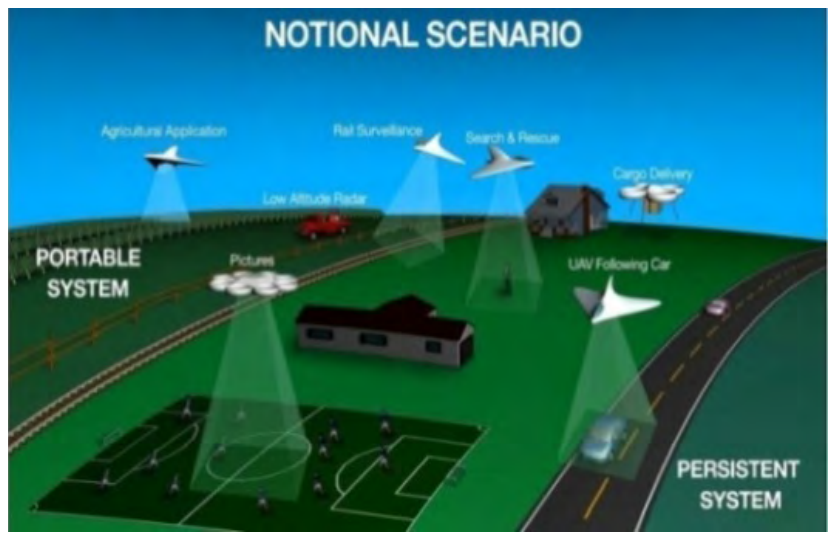

Figure 2. The Air traffic services.

All the parameters defined in the queuing problem are well explained in the application. They are as follows.

\subsection{MULTI ESSENTIAL SERVICES (STAGE 1)}

\section{STAGE I}

Flight data benefit, which offers data helpful for the sheltered and successful holding of flights; the pilot can get data about the landing and takeoff timings of different flights. They need to mindful of the climate investigation report of every single moment send by the radar frameworks. 


\section{STAGE II}

Flight data benefit, which offers data helpful for the sheltered and successful hold Alerting administration, which bears administrations to all known air ships. The pilots will get the explicit data with respect to hazardous situations. On the off chance that there is no such occurrence, it used to send one leeway message to all the air create for further procedure.

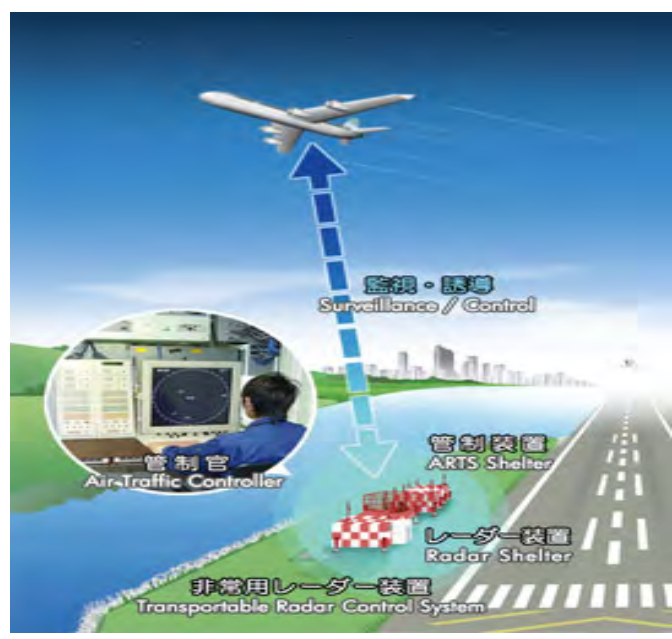

Figure 3. Alerting Administration.

\subsection{INTERRUPTION OCCURRENCE}

Air traffic controllers at every office are bolstered by various pros, for example, meteorologists, traffic the executive's experts. Subsequently, if there any correspondence connects mistake between these pros may cause enormous harm/issue to the flying machines. The showcase and control comforts utilized by controllers probably won't be at the great condition. This framework incorporates PC frameworks of changing vintages, complex voice and information exchanging gear, radio and microwave transmission frameworks, nearby and remote-found radio and radar frameworks, and also there might be a few issues in ecology and electric power molding and reinforcement frameworks, which are required by the hardware.

\subsection{REPAIR WORKS CARRIED OUT IN REPAIR PROCESS}

Repair of aging display computers. The replacement of frequency of outages involving the aging IBM 9020E display channel complex equipment. Replacement of Other recent 
equipment outages involving power systems and communications equipment. To rectify Lack of controller proficiency with the direct access radar channel (DARG) /Standalone mode of the backup computer system. Software error can be rectified by debugging the prolonged coding. Hardware repairs can be rectified by the replacement of some good conditioned equipment. Re alters the wiring connections, cables to avoid outages. Some equipment reassembly may also take place at this stage.

\subsection{COMPULSORY VACATION (REGULAR MAINTENANCE WORK)}

Any time the system is functioning with failed elements, technicians are working to make emergency repairs. Often, technicians work around the clock until repairs are complete. This restricts their ability to comprehend monotonous maintenance tasks and less-serious repairs. The Safety Boardconfirmed three basic, recurring problems distressing air route facilities: (1) Thewell-publicized age of the kit, predominantly the IBM 9020E computer and some related modules, contributes to the existing difficulties. It is hard to maintain, old system with brittle wiring, thousands of difficult-to-repair special circuit boards, and a nearly total lack of direct manufacturer support. Whenever the framework is working with fizzled components, professionals are attempting to make crisis fixes. Frequently, experts work nonstop until the point when fixes are finished. This confines their capacity to complete repetitive support undertakings and less-genuine fixes. The Safety Board affirmed three essential, repeating issues troubling air course offices:

The all-around broadcasted age of the unit, dominatingly the IBM 9020E PC and some related modules, adds to the current challenges. It is difficult to keep up, old framework with fragile wiring, a large number of hard to-fix exceptional circuit sheets, and an about the aggregate absence of direct maker bolster.

Facility reinforcement control hand-off frameworks are additionally intensifying at an expanding rate. This is by all accounts a more critical issue than 9020E disappointments, since it disturbs all electrically fueled frameworks in an office. Communication joins both radio correspondences to airplane and landline interchanges between ATC offices, are likewise confronting the issues at a rate that worries both the Safety Board and the FAA. 


\subsection{OPTIONAL EXTENDED VACATION}

Whenever the framework is working with fizzled components, experts are attempting to make crisis fixes. Regularly, specialists work nonstop until the point that fixes are finished. This confines their capacity to far reaching dull upkeep errands and less-genuine fixes. The Safety Board affirmed three fundamental, repeating issues troubling air course offices: 1) the very much exposed age of the unit, dominatingly the IBM 9020E PC and some related modules, add to the current challenges. It is difficult to keep up, old framework with fragile wiring, a large number of hard to-fix exceptional circuit sheets, and an almost add up to absence of direct producer bolster. 2) Facility reinforcement control transfer frameworks are additionally compounding at an expanding rate.

\subsection{STANDBY SERVER DURING EXTENDED VACATION}

In the event that either the Host framework or the presentation PC flops (or if both come up short), controllers must rely upon on DARC. In case of a presentation PC blackout, DARC passes on related radar and flight plan data to controller PVDs trusting on the Host to movement flight plan data. This method of activity is alluded to as "DARC/Host," and is like task under the essential framework; be that as it may, a few structures are not realistic to controllers. Five structures that are not available amid DARC/Host activity include:

Conflict alarm, a PC undermining that protected flying machine partition has been endangered. Minimum safe height cautioning (MSAW), a PC cautioning that a flying machine is working underneath a preset least elevation. Mode-C interloper alarm, a PC cautioning that an unmanaged airplane is working in the airspace. Remove reference pointer, a moving 5-mile ring around flying machine focuses on that is utilized as a partition helper.

Any time the system is functioning with failed elements, technicians are working to make emergency repairs. Often, technicians work around the clock until repairs are complete. This restricts their ability to comprehend monotonous maintenance tasks and less-serious repairs. The Safety Board confirmed three basic, recurring problems distressing air route facilities: (1) The well-publicized age of the kit, predominantly the IBM 9020E computer and some related modules, contributes to the existing difficulties. It is hard to maintain, 
old system with brittle wiring, thousands of difficult-to-repair special circuit boards, and a nearly total lack of direct manufacturer support. Whenever the framework is working with fizzled components, professionals are attempting to make crisis fixes. Frequently, experts work nonstop until the point when fixes are finished. This confines their capacity to complete repetitive support undertakings and less-genuine fixes. The Safety Board affirmed three essential, repeating issues troubling air course offices:

\section{QUEUEING THEORY APPROACH TO AIRCRAFT SERVICE PROBLEM}

The above characterized Queuing issue (Air creates issue) is disentangled by using the supplementary variable technique. For every one of the organization time, remain by server escape time and fix time valuable variables are used. Unwavering state line gauge dispersal and the different execution measure are settled. Numeral outline legitimizes the model and the graphical depiction gives a sensible picture about the decisions to be taken before the startup of the organization. To deteriorate the issue of clog in the air make benefits, an obvious endorsement is rendered toward the end, by strategies for looking at the numerical results and graphical examination of the model.

\section{NON MARKOVIANQUEUEING PROBLEM}

The Queuing issue is as per the following: This examination researches a non Markovian lining issue in which the administration is rendered in two phases. Clients touch base in clumps pursues a Poisson appropriation. Administration time pursues a general dissemination. After the consummation of the second phase of administration, the server goes for a mandatory get-away, if in need the server has the choice go take an all-encompassing excursion. Here excursion in the sense speaks to the period amid which the support works to be done in the server. Likewise, benefit hinders aimlessly amid the season of administration. All things considered, circumstances, it is unavoidable. In this lining issue, separately happens amid the second phase of administration and accepted to happen as per a Poisson stream. Likewise, to evade the blockage, amid the season of discretionary broadened get-away, a remain by the server is given. At whatever point the server intrudes on, it is sent to fix process promptly immediately. For the above characterized Queuing model, by methods for strengthening 
the variable system, we acquire the enduring state results in express and shout for regarding the likelihood producing capacities for the quantity of clients approve the model. It gives a reasonable pondered the theoretical investigation of Queuing hypothesis.

\section{SCIENTIFIC DEPICTION OF THE MODEL}

Consumers enter in clusters pursues a Poisson procedure. Let $\lambda p_{i} d t$, i varies from 1 to $\mathrm{n}$, is the probability value which denotes the arrival of $\mathrm{i}$ customers in cluster. Here $0 \leq p_{i} \leq 1$ and $\sum_{i=1}^{n} p_{i}=1$ and $\lambda>0$ is the mean landing rate of the batch. Service time follows general distribution. $\int_{0}^{\infty} S_{n}^{(a)}(x) d x, \int_{0}^{\infty} S_{n}^{(b)}(x) d x, \int_{0}^{\infty} B_{n}^{(1)}(x) d x, \int_{0}^{\infty} B_{n}^{(2)}(x) d x$ and $\int_{0}^{\infty} L_{n}(x) d x$ refers to probability that there are $\mathrm{n}$ customers in the queue and the server is in essential service, optional service, compulsory vacation, optional extended vacation and revamp process respectively. Essential service, optional service, compulsory vacation, optional extended vacation and revamp process follows general distribution with distribution function $Q_{a}(s), Q_{b}(s), R_{q 1}(s), R_{q 2}(\mathrm{~s})$ and $L(s)$ and corresponding density function $q_{a}(s), q_{b}(s), r_{q 1}(s), r_{q 2}(s)$ and $l(s)$ repectively. Also $\xi_{a}(x)$ and $\xi_{b}(x)$ be the contingent likelihood of culmination of first fundamental service and flexible administration amid the interim $(x, x+d x)$, given that the slipped time by time is $\mathrm{x}$, so that $\xi_{a}(x)=\frac{q_{a}(x)}{1-\overline{Q_{a}}(x)}$, $q_{a}(s)=\xi_{a}(s) e^{-\int \xi_{a}(x) d x}$ and $\xi_{b}(x)=\frac{q_{b}(x)}{1-\overline{Q_{b}}(x)}, q_{b}(s)=\xi_{b}(s) e^{-\int^{f} \xi_{b}(x) d x}$. Similarly for compulsory vacation and optional extended vacation, we have $\gamma(x)=\frac{r_{q 1}(x)}{1-R_{q 1}(x)}, r_{q 1}(s)=\gamma(s) e^{-\int_{0}^{f} \gamma(x) d x}$ and $\delta(x)=\frac{r_{q 2}(x)}{1-\bar{R}_{q 2}(x)}, \quad r_{q 2}(s)=\delta(s) e^{-f^{-} \delta(x) d x}$. The concept of standby server acts during the optional extended vacation period and it follows exponential distribution with parameter additionally the fix time (repair time) pursues general dispersion. So $\varepsilon(x)=\frac{l(x)}{1-\bar{L}(x)} \quad, l(s)=\epsilon(s) e^{-\int_{0}^{f} \varepsilon(x) d x}$

\section{"The issue is delineated by birth and death process by techniques for Persevering state conditions."}

\section{GOVERNING EQUATIONS OF THE QUEUEING SYSTEM IN STEADY STATE}

To start with, the generating function in terms of probability is given as follows:

$$
\begin{gathered}
S_{n}^{a}(x, z)=\sum_{n=0}^{\infty} f^{n} S_{n}^{a}(x), S_{n}^{b}(x, z)=\sum_{n=0}^{\infty} f^{n} S_{n}^{b}(x) B_{q}^{(1)}(x, f)=\sum_{n=0}^{\infty} z^{n} B_{n}^{(1)}(x) \\
B_{q}^{(2)}(x, f)=\sum_{n=0}^{\infty} z^{n} B_{n}^{(2)}(x), \mathrm{L}(x, f)=\sum_{n=0}^{\infty} z^{n} L_{n}(x)
\end{gathered}
$$


According to the above assumption and description, the following steady state equations are derived:

$$
\begin{gathered}
\frac{\partial}{\partial x} S_{n}^{(a)}(x)=-\left(\varphi+\xi_{a}(x)\right) S_{n}^{(a)}(x)+\varphi \sum_{j=1}^{n} p_{i} S_{n-j}^{(a)}(x) \\
\frac{\partial}{\partial x} S_{0}^{(a)}(x)+\left(\varphi+\xi_{a}(x)\right) S_{0}^{(a)}(x)=0 \\
\frac{\partial}{\partial x} S_{n}^{(b)}(x)=-\left(\varphi+\xi_{b}(x)+\omega\right) S_{n}^{(b)}(x)+\varphi \sum_{j=1}^{n} p_{i} S_{n-j}^{(b)}(x) \\
\frac{\partial}{\partial x} S_{0}^{(b)}(x)+\left(\varphi+\xi_{b}(x)+\omega\right) S_{0}^{(b)}(x)=0 \\
\frac{\partial}{\partial x} B_{n}^{(1)}(x)+(\varphi+\gamma(x)) B_{n}^{(1)}(x)=\sum_{j=1}^{n} p_{i} B_{n-j}^{(1)}(x) \\
\frac{\partial}{\partial x} B_{0}^{(1)}(x)+(\varphi+\gamma(x)) B_{0}^{(1)}(x)=0 \\
(x)+(\varphi+\delta(x)+\tau) B_{n}^{(2)}(x)=\varphi \sum_{j=1}^{n} p_{i} B_{n-j}^{(2)}(x)+\tau B_{n+1}^{(2)}(x) \\
\frac{\partial}{\partial x} B_{0}^{(2)}(x)+(\varphi+\delta(x)+\tau) B_{0}^{(2)}(x)=\tau B_{1}^{(2)}(x) \\
\frac{\partial}{\partial x} L_{n}(x)+(\varphi+\epsilon(x)) L_{n}(x)=\sum_{j=1}^{n} p_{i} L_{n-j}(x) \\
\frac{\partial}{\partial x} L_{0}(x)+(\varphi+\epsilon(x)) L_{0}(x)=0
\end{gathered}
$$

Boundary conditions:

$$
\begin{gathered}
Q D=\int_{0}^{\infty} L_{0}(x) \varepsilon(x) d x+(1-c) \int_{0}^{\infty} B_{0}^{(1)}(x) \gamma(x) d x+\int_{0}^{\infty} B_{0}^{(2)}(x) \delta(x) d x \\
S_{n}^{(a)}(0)=\int_{0}^{\infty} B_{n+1}^{(2)}(x) \delta(x) d x+\int_{0}^{\infty} L_{n+1}(x) \varepsilon(x) d x+\varphi p_{n+1} D+(1-c) \int_{0}^{\infty} B_{n+1}^{(1)}(x) \gamma(x) d x \\
S_{n}^{(b)}(0)=\int_{0}^{\infty} S_{n}^{(a)}(x) \xi_{a}(x) d x \\
B_{n}^{(1)}(0)=\int_{0}^{\infty} S_{n}^{(b)}(x) \xi_{b}(x) d x, \quad x \geq 0 \\
B_{n}^{(2)}(0)=c \int_{0}^{\infty} B_{n}^{(1)}(x) \gamma(x) d x, \quad x \geq 0
\end{gathered}
$$




$$
L_{n}(0)=\omega \int_{0}^{\infty} S_{n-1}^{(b)}(x) d x=\omega S_{n-1}^{(b)}, \quad L_{0}(0)=0
$$

\section{QUEUE SIZE DISTRIBUTION AT A RANDOM EPOCH}

Usage of Supplementary variable method:

We multiply (1) by $f^{n}$ and sum over $n$ from 1 to $\infty$ and add it to (2) results as follows:

$$
\frac{\partial}{\partial x} S_{q}^{(a)}(x, f)+\left(\varphi-\varphi P(f)+\xi_{a}(x)\right) S_{q}^{(a)}(x, f)=0
$$

Applying the similar procedure for the remaining parameters, we have

$$
\begin{gathered}
\frac{\partial}{\partial x} S_{q}^{(b)}(x, f)+\left(\varphi-\varphi P(f)+\xi_{b}(x)+\omega\right) S_{q}^{(b)}(x, f)=0 \\
\frac{\partial}{\partial x} B_{q}^{(1)}(x, f)+(\varphi-\varphi P(f)+\gamma(x)) B_{q}^{(1)}(x, f)=0 \\
\frac{\partial}{\partial x} B_{q}^{(2)}(x, f)+\left(\varphi-\varphi P(f)+\gamma(x)+\tau-\frac{\tau}{f}\right) B_{q}^{(2)}(x, f)=0 \\
\frac{\partial}{\partial x} L_{q}(x, f)+(\varphi-\varphi P(f)+\epsilon(x)) L_{q}(x, f)=0
\end{gathered}
$$

Multiplying (12a) by $f^{n+1}$ and summing over $n$ from 0 to $\infty$ and using (11), we get

$$
f S_{q}^{(a)}(0, f)=\int_{0}^{\infty} B_{q}^{(2)}(x, f) \delta(x) d x+\int_{0}^{\infty} L_{q}(x, f) \varepsilon(x) d x+(1-c) \int_{0}^{\infty} B_{q}^{(1)}(x, f) \gamma(x) d x+\varphi D(P(z)
$$

To find the R.H.S of (21), we proceed as follows

Next multiply (12b) by $f^{n}$ and summing over $n$ from 0 to $\infty$ to obtain

$$
\begin{gathered}
S_{q}^{(b)}(0, f)=\int_{0}^{\infty} S_{q}^{(a)}(x, f) \xi_{a}(x) d x \\
B_{q}^{(1)}(0, f)=\int_{0}^{\infty} S_{q}^{(b)}(x, f) \xi_{b}(x) d x \\
B_{q}^{(2)}(0, f)=c \int_{0}^{\infty} B_{q}^{(1)}(x, f) \gamma(x) d x \\
L_{q}(0, f)=\omega f \int_{0}^{\infty} S_{q}^{(b)}(x, f) d x=\omega f S_{q}^{(b)}(f)
\end{gathered}
$$

Integrating (16) from 0 tox yields 


$$
S_{q}^{(a)}(x, f)=S_{q}^{(a)}(0, f) e^{-(\varphi-\varphi P(f)) x-\int_{0}^{x} \xi_{a}(t) d t}
$$

Where $S_{q}^{(a)}(0, f)$ is given by $(21)$

Integrating (26) by parts with respect to yields

$$
S_{q}^{(a)}(f)=\frac{S_{q}^{(a)}(0, f)\left(1-Q_{a}(\varphi-\varphi P(f))\right)}{\varphi-\varphi P(f)}
$$
Where $\overline{Q_{a}}=\int_{0}^{\infty} e^{-(\varphi-\varphi P(f)) x} d Q_{a}(x)$ is the Laplace Stieltje's transform of the service time
$Q_{a}(x)$.

Multiply both sides of (26) by $\xi_{a}(x)$ and integrating over $x$ we get

$$
\int_{0}^{\infty} S_{q}^{(a)}(x, f) \xi_{a}(x) d x=S_{q}^{(a)}(0, f) \overline{Q_{a}}(\varphi-\varphi P(f))
$$

Similar procedure to be carried out for $(17)-(20)$, we get

$$
\begin{gathered}
S_{q}^{(b)}(f)=\frac{S_{q}^{(b)}(0, f)\left(1-Q_{b}(\varphi-\varphi P(f)+\omega)\right)}{\varphi-\varphi P(f)+\omega} \\
\int_{0}^{\infty} S_{q}^{(b)}(x, f) \xi_{b}(x) d x=S_{q}^{(a)}(0, f) \overline{Q_{a}}(\varphi-\varphi P(f)) \overline{Q_{b}}(\varphi-\varphi P(f)+\omega)
\end{gathered}
$$

For the process of compulsory vacation,

$$
\begin{gathered}
B_{q}^{(1)}(f)=\frac{B_{q}^{(1)}(0, f)\left(1-R_{q_{1}}(\varphi-\varphi P(f))\right)}{\varphi-\varphi P(f)} \\
\int_{0}^{\infty} B_{q}^{(1)}(x, f) \gamma(x) d x=S_{q}^{(a)}(0, f) \overline{Q_{a}}(\varphi-\varphi P(f)) \overline{Q_{b}}(\varphi-\varphi P(f)+\omega) \overline{R_{q_{1}}}(\varphi-\varphi P(f))
\end{gathered}
$$

For optional extended vacation,

$$
\begin{gathered}
B_{q}^{(2)}(f)=\frac{B_{q}^{(2)}(0, f)\left(1-\overline{R_{q_{2}}}\left(\varphi-\varphi P(f)+\tau-\frac{\tau}{f}\right)\right)}{\varphi-\varphi P(f)+\tau-\frac{\tau}{f}} \\
\int_{0}^{\infty} B_{q}^{(2)}(x, f) \delta(x) d x=c S_{q}^{(a)}(0, f) \overline{Q_{a}}(\varphi-\varphi P(f)) \overline{Q_{b}}(\varphi-\varphi P(f)+\omega) \overline{R_{q_{1}}}(\varphi-\varphi P(f)) \overline{R_{q_{2}}}(\varphi
\end{gathered}
$$


For revamp process,

$$
\begin{gathered}
L_{q}(f)=\frac{L_{q}(0, f)(1-L(\varphi-\varphi P(f)+\omega))}{\varphi-\varphi P(f)+\omega} \\
\int_{0}^{\infty} L_{q}(x, f) \varepsilon(x) d x=\frac{\omega f\left(1-\overline{Q_{b}}(\varphi-\varphi P(f)+\omega)\right)\left(\bar{L}(\varphi-\varphi P(f)+\omega) S_{q}^{(a)}(0, f) \overline{Q_{a}}(\varphi-\varphi P(f))\right)}{(\varphi-\varphi P(f)+\omega)}
\end{gathered}
$$

Using (32), (34), (36) in (21), we get:

$$
S_{q}^{(a)}(0, f)=-\frac{D(\varphi-\varphi P(f))}{f-\overline{Q_{a}}(\varphi-\varphi P(f))\left\{\frac{1-\bar{Q}(\varphi-\varphi P(f)+\omega)}{\varphi-\varphi P(f)+\omega}\right\} L(\varphi-\varphi P(f)+\omega) \omega f-\overline{Q_{a}}(\varphi-\varphi P(f)) \overline{Q_{b}}(\varphi-\varphi P(f)+\omega) \overline{R_{q_{1}}}(\varphi-\varphi P(f))\left(c \overline{R_{q_{2}}}(\varphi\right.}
$$

Substituting (37) in (27),(29),(31),(33) we get:

$$
\begin{gathered}
S_{q}^{(a)}(f)=\frac{S_{q}^{(a)}(0, f)\left(1-\overline{Q_{a}}(\varphi-\varphi P(f))\right)}{\varphi-\varphi P(f)} \\
S_{q}^{(b)}(f)=\frac{S_{q}^{(b)}(0, f)\left(1-\overline{Q_{b}}(\varphi-\varphi P(f)+\omega)\right)}{\varphi-\varphi P(f)+\omega} \\
B_{q}^{(1)}(f)=\frac{B_{q}^{(1)}(0, f)\left(1-R_{q_{1}}(\varphi-\varphi P(f))\right)}{\varphi-\varphi P(f)} \\
B_{q}^{(2)}(f)=\frac{B_{q}^{(2)}(0, f)\left(1-R_{q_{2}}\left(\varphi-\varphi P(f)+\tau-\frac{\tau}{f}\right)\right)}{\varphi-\varphi P(f)+\tau-\frac{\tau}{f}} \\
L_{q}(f)=\frac{L_{q}(0, f)(1-L(\varphi-\varphi P(f)+\omega))}{\varphi-\varphi P(f)+\omega}
\end{gathered}
$$

\section{PROBABILITY GENERATING FUNCTION OF THE QUEUE LENGTH}

Let $A^{* *}(f)$ be the likelihood producing capacity of the line length regardless of what the framework is,

$$
\text { (i.e.) } A^{* *}(f)=S_{q}^{(a)}(f)+S_{q}^{(b)}(f)+B_{q}^{(1)}(f)+B_{q}^{(2)}(f)+L_{q}(f)
$$

Thus adding (26),(29),(31),(33) and (35) we get:

$$
A^{* *}(z)=\frac{D\left\{\left(-\left(1-\overline{Q_{a}}(\varphi-\varphi P(f))\right)-\left(1-\overline{R_{q_{1}}}(\varphi-\varphi P(f))\right) \overline{Q_{a}}(\varphi-\varphi P(f)) \overline{Q_{b}}(\varphi-\varphi P(f)+\omega)+\omega f \overline{Q_{a}}(\varphi-\varphi P(f))\left\{\frac{1-Q_{b}(\varphi-\varphi P(f)+\omega)}{\varphi-\varphi P(f)+\omega}\right\} \bar{L}\right.\right.}{D(z)}
$$


Where:

$D(z)=f-\overline{Q_{a}}(\varphi-\varphi P(f))\left\{\frac{1-Q_{b}(\varphi-\varphi P(f)+\omega)}{\varphi-\varphi P(f)+\omega}\right\} \bar{L}(\varphi-\varphi P(f)+\omega) \omega f-\overline{Q_{a}}(\varphi-\varphi P(f)) \overline{Q_{b}}(\varphi-\varphi P(f)+\omega)$

\section{IDLE TIME AND UTILIZATION FACTOR}

The normalization condition $A^{* *}(1)+D=1 \quad(44)$.

Gives out the idle time and hence the time spent by the server for the service.

L'Hopital's rule is applied on (43) due to its determinant form to achieve

$$
\lim _{z \rightarrow 1} A^{* *}(z)=\frac{\varphi\left[1+E\left(Q_{a}\right)+E\left(R_{q_{1}}\right)\right]+\left(1-Q_{b}(\omega)\right) E(L)}{1-E\left(Q_{a}\right) \varphi\left(1-\overline{Q_{b}}(\omega)\right)-E\left(Q_{b}\right) \omega-E(L) \varphi\left(1-\overline{Q_{b}}(\omega)\right)-\left(1-\overline{Q_{b}}(\omega)\right)+\varphi\left[E\left(Q_{a}\right)+E\left(Q_{b}\right)+E\left(R_{q_{1}}\right)\right]-(\tau-\varphi) c E\left(R_{q_{2}}\right)}
$$

Now using (45) and normalizing condition we get

$$
D=\frac{1-E\left(Q_{a}\right) \varphi\left(1-\overline{Q_{b}}(\omega)\right)-E\left(Q_{b}\right) \omega-E(L) \varphi\left(1-\overline{Q_{b}}(\omega)\right)-\left(1-\overline{Q_{b}}(\omega)\right)+\varphi\left[E\left(Q_{a}\right)+E\left(Q_{b}\right)+E\left(R_{q}\right)\right]-(\tau-\varphi) c E}{\left[\varphi\left[1+E\left(Q_{a}\right)+E\left(R_{q}\right)\right]+\left(1-\overline{Q_{b}}(\omega)\right) E(L)\right]\left\{1-E\left(Q_{a}\right) \varphi\left(1-\overline{Q_{b}}(\omega)\right)-E\left(Q_{b}\right) \omega-E(L) \varphi\left(1-\overline{Q_{b}}(\omega)\right)-\left(1-\overline{Q_{b}}(\omega)\right)+\varphi\left[E\left(Q_{a}\right)+E(Q\right.\right.}
$$

Also from the above, the utilization factor can be calculated, using $\rho=1-D$

\section{EXECUTION MEASURES OF THE LINING SYSTEM}

To find $L_{q}$, where $L_{q}=\frac{d}{d z} A^{* *}(z) \mid z=1$

As it takes indeterminate form as z tends to 1, L 'Hopital's rule is applied. Hence

$$
\begin{gathered}
L_{q}=\lim _{z \rightarrow 1} \frac{D^{\prime}(z) N^{\prime \prime}(z)-N^{\prime}(z) D^{\prime \prime}(z)}{2\left(D^{\prime}(z)\right)^{2}} \\
D^{\prime}(1)=1-E\left(Q_{a}\right) \varphi\left(1-\overline{Q_{b}}(\omega)\right)-E\left(Q_{b}\right) \omega-E(L) \varphi\left(1-\overline{Q_{b}}(\omega)\right)-\left(1-\overline{Q_{b}}(\omega)\right)+\varphi \\
D^{\prime \prime}(1)=-2 \varphi E\left(Q_{a}\right) E\left(Q_{b}\right) \omega-3 \varphi\left(1-\overline{Q_{b}}(\omega)\right) E(L)-2 \varphi E\left(Q_{a}\right)\left(1-\overline{Q_{b}}(\omega)\right)-E\left(Q_{b}^{2}\right) \varphi \\
N^{\prime}(1)=\varphi\left[1+E\left(Q_{a}\right)+E\left(R_{q_{1}}\right)\right]+\left(1-\overline{Q_{b}}(\omega)\right) E(L) \\
N^{\prime \prime}(1)=\varphi^{2}\left[E\left(Q_{a}^{2}\right)+\overline{Q_{b}}(\omega)\left(E\left(R_{q_{1}}^{2}\right)+2 E\left(R_{q_{1}}\right) E\left(Q_{a}\right)+\frac{c}{\varphi}\right)+2 E\left(R_{q_{1}}\right) E\left(Q_{b}\right)\right]-\varphi E
\end{gathered}
$$


The above derivatives are substituted in (47) we obtain $L_{q}$ in closed form. Further the other performance measures of the defined queuing model are found using Little's formula $W_{q}=\frac{L_{q}}{\lambda} \quad,=\frac{L}{\lambda}, L=L_{q}+\rho$.

\section{NUMERICAL ILLUSTRATION}

We portray a numerical point of reference with the ultimate objective to see the effect and authenticity of our results of the particular parameters used in our model, to be explicit reneging rate, separate rate and likelihood of culmination of fix rate on the utilization factor and execution extents of the coating model. The estimations of the parameters are assembled with the ultimate objective that the unfaltering condition isn't harmed.

Assume service time follows an exponential distribution:

$$
\begin{aligned}
& E\left(Q_{a}\right)=\frac{1}{\xi_{a}}, E\left(Q_{a}^{2}\right)=\frac{2}{\xi_{a}^{2}}, E(L)=\frac{1}{\varepsilon}, \overline{Q_{b}}(\omega)=\frac{\xi_{b}}{\xi_{b}+\omega}, E\left(L^{2}\right)=\frac{2}{\varepsilon^{2}}, \\
& {\overline{Q_{b}}}^{\prime}(\omega)=\frac{-\xi_{b}}{\left(\xi_{b}+\omega\right)^{2}},{\overline{Q_{b}}}^{\prime}(\omega)=\frac{2 \xi_{b}}{\left(\xi_{b}+\omega\right)^{3}}, E\left(R_{q_{1}}\right)=\frac{1}{\gamma}, E\left(R_{q_{2}}\right)=\frac{1}{\delta}, \\
& E\left(R_{q_{1}}^{2}\right)=\frac{2}{\gamma^{2}}, E\left(R_{q_{2}}^{2}\right)=\frac{2}{\delta^{2}}, \xi_{a}=4, \xi_{b}=5, \omega=2, \gamma=3.5, \delta=2.5 \\
& , \epsilon=5, \tau=3, \varphi=3, c=0.5
\end{aligned}
$$

Case (I): Table 1 demonstrate that for steady estimations of all the parameters and expanding the arrival rate $\varphi$, demonstrates an expansion in all the executions estimates like $L_{q^{\prime}} L, W_{q^{\prime}}$ $W$. Additionally, it prompts a contraction in usage factor and at the same time an increase in idle time.

Table 1. Effect of Variation Of Arrival Rate $\varphi=1,2.5,3,4,5,6,7$.

\begin{tabular}{|c|c|c|c|c|c|}
\hline $\boldsymbol{Q}$ & $\boldsymbol{\rho}$ & $\boldsymbol{L}_{\boldsymbol{q}}$ & $\boldsymbol{W}_{\boldsymbol{Q}}$ & $\boldsymbol{L}$ & $\boldsymbol{W}$ \\
\hline 0.3623 & 0.6377 & 0.5463 & 0.1821 & 1.184 & 0.3947 \\
\hline 0.3782 & 0.6218 & 0.8571 & 0.2143 & 1.4789 & 0.3697 \\
\hline 0.3875 & 0.6125 & 1.0928 & 0.2186 & 1.7053 & 0.3411 \\
\hline 0.3935 & 0.6065 & 1.2994 & 0.2166 & 1.9059 & 0.3177 \\
\hline 0.4071 & 0.5929 & 1.4398 & 0.2057 & 2.0327 & 0.2904 \\
\hline
\end{tabular}




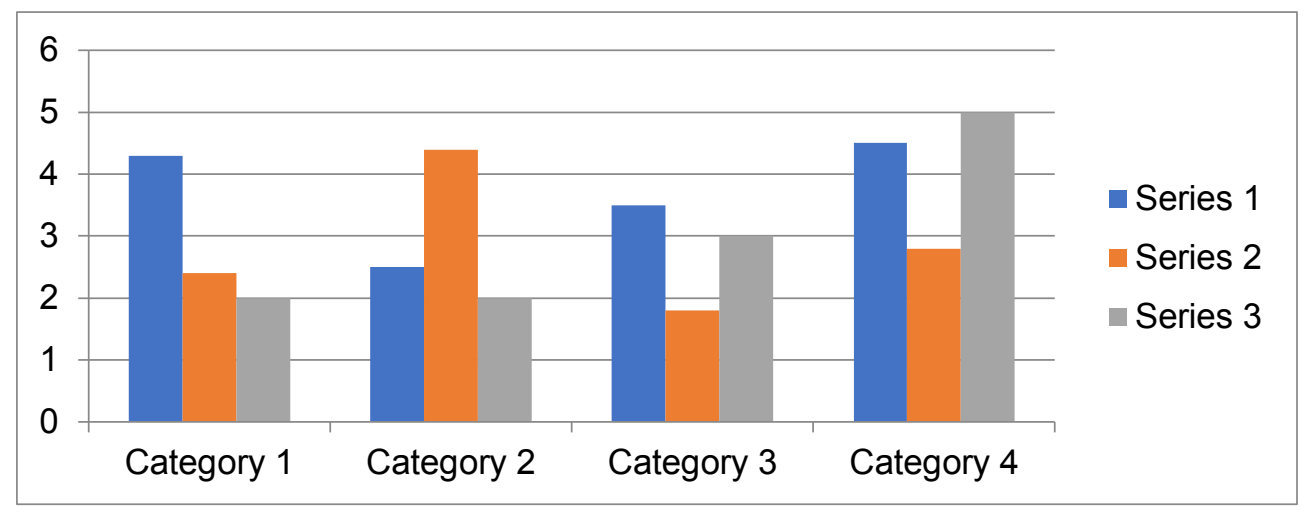

Graphic 1. Variation of $\varphi$.

Case (II): From Table 2, It is clear that if the probability in completion of repair rate increases, it leads to a decrease in all the performance measures. Since the repair gets completed sooner, the idle time of the server increases, and the time spent by the server for service get expanded.

Table 2. Effect of Variation of Repair Rate $\epsilon=5,6,7,8,9$.

\begin{tabular}{|c|c|c|c|c|c|}
\hline $\boldsymbol{Q}$ & $\boldsymbol{\rho}$ & $\boldsymbol{L}_{\boldsymbol{q}}$ & $\boldsymbol{W}_{\boldsymbol{Q}}$ & $\boldsymbol{L}$ & $\boldsymbol{W}$ \\
\hline 0.2822 & 0.7178 & 0.2080 & 0.0693 & 0.9258 & 0.3086 \\
\hline 0.2857 & 0.7143 & 0.1610 & 0.0537 & 0.8753 & 0.2918 \\
\hline 0.2883 & 0.7117 & 0.1310 & 0.0437 & 0.8427 & 0.2809 \\
\hline 0.2902 & 0.7098 & 0.1105 & 0.0368 & 0.8203 & 0.2734 \\
\hline 0.2916 & 0.7084 & 0.0956 & 0.0319 & 0.8040 & 0.2680 \\
\hline
\end{tabular}

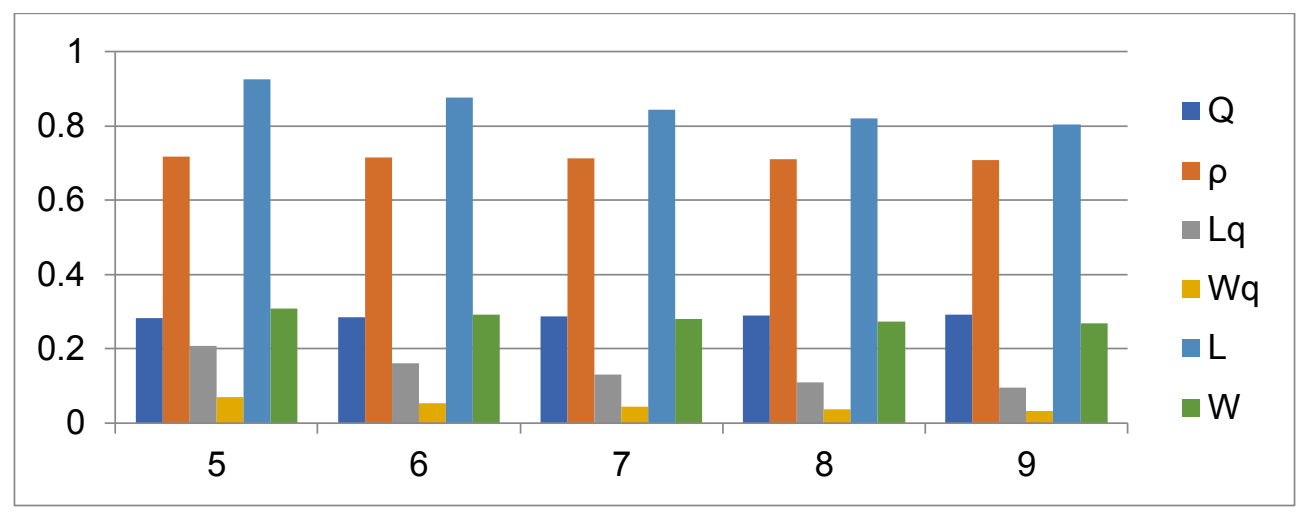

Graphic 2. Variation of $\varepsilon$. 
Case (III): Table 3 indicates that an increase in Reneging rate $\tau$ makes all the performance measures to decrease. Additionally, it prompts an expansion in usage factor and at the same time a decrease out of idle time.

Table 3. Effect of Variation of Standby Server Rate $\tau=3,3.5,4,4.5,5,5.5$.

\begin{tabular}{|c|c|c|c|c|c|}
\hline $\boldsymbol{Q}$ & $\boldsymbol{\rho}$ & $\boldsymbol{L}_{\boldsymbol{q}}$ & $\boldsymbol{W}_{\boldsymbol{Q}}$ & $\boldsymbol{L}$ & $\boldsymbol{W}$ \\
\hline 0.3709 & 0.6291 & 0.5819 & 0.1940 & 1.2110 & 0.4037 \\
\hline 0.3623 & 0.6377 & 0.5461 & 0.1820 & 1.1838 & 0.3946 \\
\hline 0.3535 & 0.6465 & 0.4822 & 0.1607 & 1.1287 & 0.3762 \\
\hline 0.3444 & 0.6556 & 0.3833 & 0.1278 & 1.0389 & 0.3463 \\
\hline 0.3350 & 0.6650 & 0.2417 & 0.0806 & 0.9067 & 0.3022 \\
\hline 0.3254 & 0.6746 & 0.0465 & 0.0155 & 0.7211 & 0.2404 \\
\hline
\end{tabular}

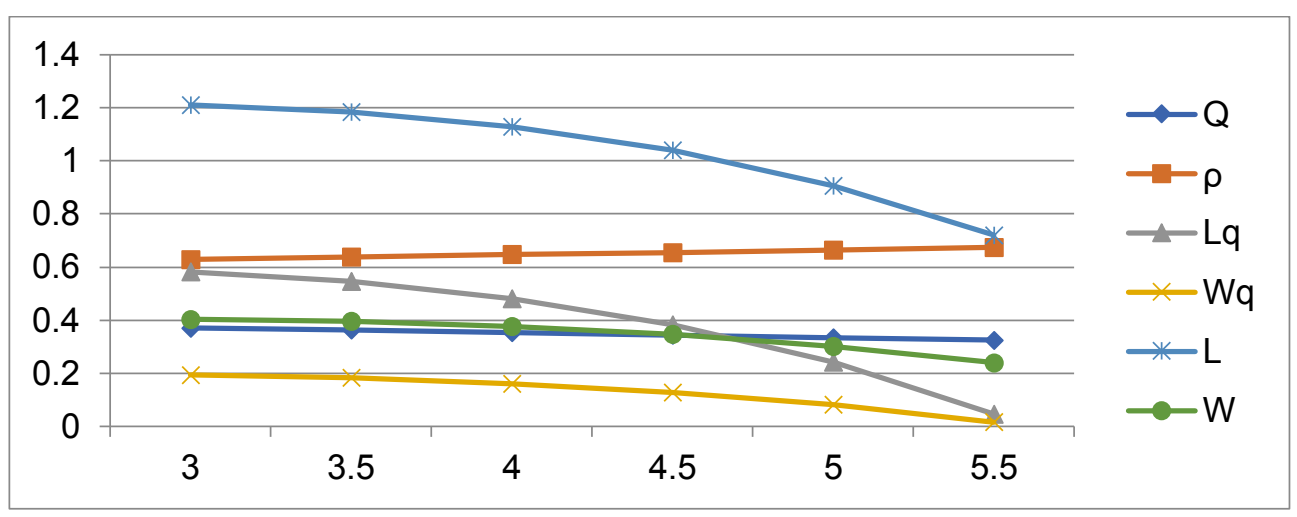

Graphic 3. Variation of $\tau$.

Case (IV): Table 4 demonstrates that for consistent estimations of all the parameters and expanding the completion of the compulsory vacation rate $\gamma$, demonstrates a contraction in all the executions estimates like $L_{q}, L, W_{q}, W$. Furthermore it prompts a development in use factor and in the meantime a decline out of inert time.

Table 4. Effect of Variation of Vacation rate $\gamma=2.5,3,4,5,6$.

\begin{tabular}{|c|c|c|c|c|c|}
\hline $\boldsymbol{Q}$ & $\boldsymbol{\rho}$ & $\boldsymbol{L}_{q}$ & $\boldsymbol{W}_{\boldsymbol{Q}}$ & $\boldsymbol{L}$ & $\boldsymbol{W}$ \\
\hline 0.3030 & 0.697 & 0.8895 & 1.5865 & 1.7965 & 0.5288 \\
\hline 0.2914 & 0.7086 & 0.7885 & 0.2628 & 1.4971 & 0.499 \\
\hline 0.2748 & 0.7252 & 0.5499 & 0.1833 & 1.2751 & 0.425 \\
\hline 0.2635 & 0.7365 & 0.3046 & 0.1015 & 1.0411 & 0.347 \\
\hline 0.2553 & 0.7447 & 0.0713 & 0.0238 & 0.8160 & 0.2720 \\
\hline
\end{tabular}




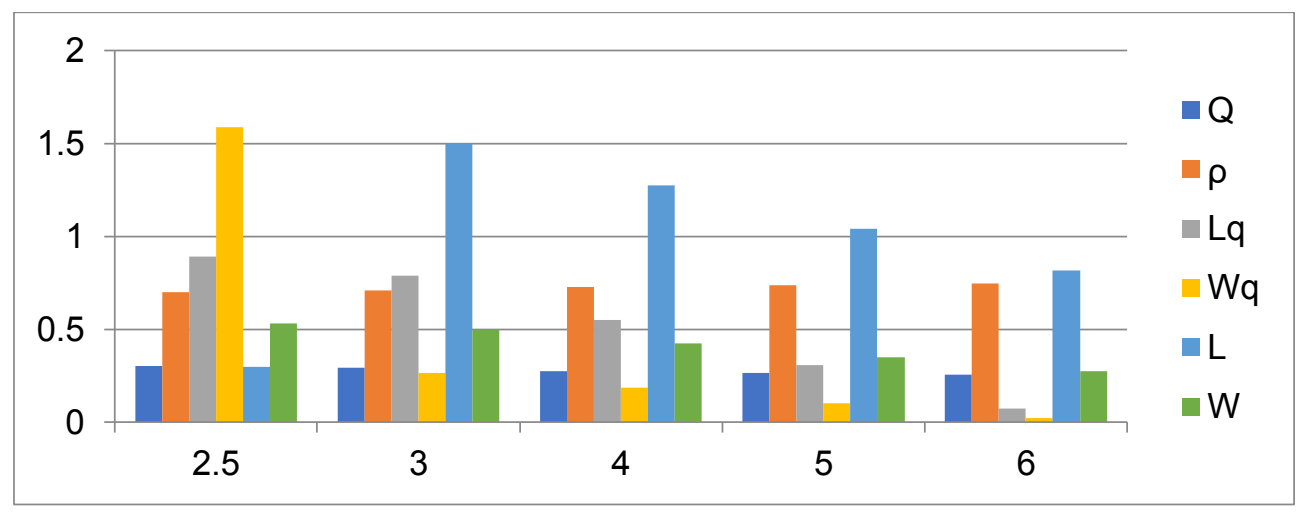

Graphic 4. Variation of $\gamma$.

\section{CONCLUSION}

The model portrayed finds its applications in various divisions of amassing organizations and correspondence frameworks. It is extraordinarily sensible to depict about stay by the server in the midst of optional extended vacation. This kind of vacation makes the server complete their help, work rely on need. As it isn't deterministic, if the upkeep work is most outrageous it can take a long journey or else short escape time. Graphical depiction pictures the model broadly and gives the reasonable results as obvious. As a future work, shying without end can be displayed. Need standard can be included. Likewise the re-try strategy can be given in stages subject to the kind of fix. Postpone process can moreover be considered before getting into a fix up process. Set up time criteria can be included in this concept of Queuing. This presentation accepts prominent employment in gathering units, correspondence structure, and movement crossing focuses, system designing and so on. 


\section{REFERENCES}

Altman, E., \& Yechiali, U. (2006). Analysis of customer's impatience in queue with server vacations. Queueing systems, 5(4), 261-279. https://doi.org/10.1007/s11134006-6134-x

Artalejo, R. J., \& Choudhury, G. (2004). Steady state analysis of an M/G/1 queue with repeated attempts and two phase service. Quality Technology and Quantitative Management,1(2), 189-199. https://doi.org/10.1080/16843703.2004.11673072

Doshi, B. T. (1986). Queueing systems with vacations-a survey. Queueing systems, 1(12), 2966. https://doi.org/10.1007/BF01149327

Keilson, J., \& Servi, L. D. (1987). The dynamics of an M/G/1 vacation model. Operations research, 35(4), 575-582. https://doi.org/10.1287/opre.35.4.575

Lee, H. W., Lee, S. S., \& Chae, K. G. (1994). Operating characteristics of M[X]/G/1 queue with N policy. Queueing system, 15(1-4), 387-399. https://doi.org/10.1007/ BF01189247

Madan, K. G. (2000). On a single server queue with two stage heterogeneous service ad deterministic server vacations. The Egyptian Statistical journal, 44(1), 39-55.

Madan, K. G., \& Abu Al-Rub, A. Z. (2004). On a single server queue with optional phase type server vacations based on exhaustive deterministic service and a single vacation policy. Applied Mathematics and Computation, 149, 723-734. https://doi.org/10.1016/ S0096-3003(03)00174-7

Madan, K. C., \& Baklizi, A. (2002). An M/G/1 queue with additional second stage service and optional re service. International journal of information and Management sciences, 16(2), 1-16. https://www.researchgate.net/publication/285743897_An_MG1_ queue_with_additional_second_stage_service_and_optical_re-service 
Maragathasundari, S., \& Balamurugan, B. (2015). A study on the performance analysis of a batch arrival queue with two stages of service Bernoulli schedule vacation extended vacation and service interruption. International journal of computer applications, 124(12), 33-37. https://www.ijcaonline.org/archives/volume124/ number12/22159-2015905695

Maragathasundari, S., \& Dhanalakshmi, K. S. (2018). Mobile adhoc networks problem- A queuing approach. International journal of communication networks and distributed systems, 21(4). https://doi.org/10.1504/IJCNDS.2018.095363

Maragathasundari, S., \& Srinivasan, S. (2012). Analysis of M/G/1 feedback queue with three stages and multiple server vacation. Applied mathematical sciences, 6(125), 6221-6240.

Maragathasundari, S., \& Srinivasan, S. (2015). A Non-Markovian Multistage Batch arrival queue with breakdown and reneging. Mathematical problems in engineering. Article ID 519579. http://dx.doi.org/10.1155/2014/519579

Maragathasundari, S., \& Srinivasan, S. (2017). Optional services in a Non Markovian Queue. International fournal of Operational Research, 28(1), 1-17. https://doi. org/10.1504/IJOR.2017.10000660

Maraghi, F. A., Madan, K. C., \& Darby-Dowman, K. (2010). Batch Arrival vacation queue with second optional service and Random Breakdowns. International Fournal of Statistical Theory and Practice, 4(1), 137-153. https://doi.org/10.1080/15598608.2010 .10411977 